\title{
Bioefficacy of Newer and Biorational Insecticides against Shoot and Fruit Borer, Earias spp. on Okra
}

\author{
Surendra Kumar Yadav, K.C. Kumawat, H.L. Deshwal", \\ Susheel Kumar" and S.V.S. Manohar*
}

\author{
Department of Entomology, S. K. N. College of Agriculture, Jobner, Jaipur, \\ Rajasthan- 303329, India \\ *Corresponding author
}

\section{A B S T R A C T}

Keywords

Bioefficacy, Insecticides, Fruit, Experiment.

Article Info

Accepted:

17 June 2017

Available Online:

10 July 2017
An experiment was conducted on bioefficacy of newer and biorational insecticides against shoot and fruit borer on okra. Efficacy of insecticides tested against Earias spp on okra indicated that the minimum per cent infestation of shoot and fruit borer was found in the treatment of indoxacarb $(1.36 \%)$ followed by spinosad $(1.75 \%)$, emamectin benzoate $(2.57 \%)$ and fipronil $(2.98 \%)$. The acetamiprid, acephate, dimethoate/ malathion and NSKE registered 3.25, 4.23, 4.81 and 5.28 per cent infestation of shoot and fruit borer and existed in moderate group of efficacy. The treatments of $B$. bassiana with 6.80 per cent infestation proved least effective followed by Btk $(6.17 \%)$.

\section{Introduction}

Okra, Abelmoschus esculentus (L.) Moench commonly known as bhindi or lady's finger (family: Malvaceae) is a popular fruit vegetable crop and said to be originated from Africa. It is an important summer and rainy season vegetable crop grown throughout the world.

In India, it is cultivated throughout the country occupying an area of 507 thousand hectares with an annual production of 5853 thousand metric tones during 2014-15 (Anonymous, 2015). In Rajasthan, it is grown in an area of 4.21 thousand hectares with an annual production to the tune of 16.64 thousand metric tonnes (Anonymous, 2015).
The crop, right from germination to harvesting is attacked by about 72 species of insect pests (Rao and Rajendran, 2003). Among them, the shoot and fruit borer ( $E$. insulana and E. vittella) is one of the most serious pests of okra. The larvae bore into the terminal growing shoots, floral buds, flowers and fruits of okra, resulting in cessation, withering and drying of infested shoots, tender leaves and heavy shedding of floral buds and flowers. The infested fruits become malformed and are rendered unfit for human consumption as well as for procurement of the seeds. The borer has been reported to cause 24.6 to 26.0 per cent damage to okra shoots (Pareek et al., 1986; Zala et al., 1999) and 40 
to 100 per cent loss to fruits (Dhawan and Sidhu, 1984; Kumawat, 1997; Shah et al., 2001; Pareek and Bhargava, 2003; Shinde et al., 2007).

The chemical control has been suggested by many workers to combat with the insect pests of okra (Samuthiravelu and David, 1991; Manjanaik et al., 2002) but due to one or other reasons, could not become panacea in protection of the crop. The use of insecticides have undoubtedly resulted in the maximum production but the proliferation of insecticides and their unilateral utilization have created many problems such as development of resistance in insect pests to insecticides, resurgence of insect pests, outbreak of secondary insect pests, insecticidal residues etc. The heavy dependence on highly toxic insecticides leads to produce toxicity hazards to environment. Keeping the point in view, field trials on management of Earias spp through different newer and biorational insecticides in okra were conducted and the results are presented.

\section{Materials and Methods}

Investigation on the bioefficacy of newer and biorational insecticides was carried out at the experimental farm, Niche Area of Excellance, Swami Keshwanand Rajasthan Agricultural University, Bikaner (Rajasthan) during kharif 2013 and 2014. The experiment was laid out in simple randomized block design (RBD) with eleven treatments including untreated control, each replicated thrice. The plot size was kept $3.0 \times 2.25 \mathrm{~m}^{2}$ keeping row to row and plant to plant distance of 45 and $30 \mathrm{~cm}$, respectively. The okra variety, Parbhani Kranti was used in experiment and was sown on $5^{\text {th }}$ July, 2013 and 2014.

Three foliar sprays of all the insecticides were given at three weeks interval. The spray was done by using knap sack sprayer. Utmost care was taken to check the drift of insecticides by putting polythene check screen around each plot at the time of spraying. The quantity of water used for plot was increased depending on the growth of the crop. The quantity of spray solutions required for full coverage in first, second and third insecticidal application were 500, 600 and 650 litres per hectare, respectively.

The shoot damage (due to shoot and fruit borer) was recorded at weekly interval while fruit damage at each picking. The yield data were recorded at each picking and were converted per hectare. The data on shoot and fruit borer, Earias spp. were recorded on five randomly selected and tagged plants throughout the crop period by visual count of the plant in which the top portion was damaged, started after two week of sowing to last picking of the fruits.

The per cent shoot infestation was calculated by counting the total number of shoots and the number of damaged shoots. In case of fruit borer, the observations were recorded on infestation of fruits both on number and weight basis at each picking starting from $13^{\text {th }}$ August, 2013 and 15 ${ }^{\text {th }}$ August, 2014 till last picking of fruits during two consecutive years. The per cent infestation of fruits on number basis was calculated by counting the infested and healthy fruits separately from selected tagged plants.

The weight of both healthy and infested fruits was taken separately and level of infestation was worked out in per cent.

The data on per cent infestation of shoots and fruits of okra (kharif, 2013, kharif, 2014 and pooled) were transformed into angular values and subjected to analysis of variance. The healthy fruits of all the pickings in each treatment were pooled together to work out the total yield. 


\section{Results and Discussion}

\section{Shoot damage by Earias spp.}

The treatment of indoxacarb with 1.36 per cent infestation of shoot was found to be the most effective followed by spinosad (1.75\%), emamectin benzoate $(2.57 \%)$ and fipronil (2.98\%) based on pooled data of Kharif 2013 and 2014 in the present investigation (Table $1)$.

Dabhi et al., (2012) reported indoxacarb @ $0.0075 \%$ had significantly superior over other treatments in controlling E. vittella in okra, corroborates with the present findings.

Shinde et al., (2011) reported spinosad 0.005 per cent followed by indoxacarb 0.01 per cent and profenophose 0.08 per cent as the most effective insecticides in controlling okra shoot and fruit borer, support the present findings. Naik and Kumar (2014) tested different insecticides and reported spinosad 45 SC @ $0.005 \%$ most effective against shoot and fruit borer (Table 2).

Patra et al., (2009) determined the field efficacy of emamectin benzoate and recorded the lowest shoot infestation of shoot and fruit borer, conform the present findings.

Parthiban et al., (2014) reported emamectin benzoate $5 \mathrm{WG}(125$ and $150 \mathrm{~g} / \mathrm{ha})$ as best insecticide and reduced more than 90 per cent population of $E$. vittella on okra corroborate the present findings.

The treatments of acetamiprid, acephate, dimethoate and NSKE registered 3.25, 4.23, 4.81 and 5.28 per cent infestation of shoot and existed in moderate group of efficacy. Das et al., (2001) evaluated the bio-efficacy of different pesticides and observed 12.42 and 14.25 infestation in acetamiprid and acephate, respectively partially support the present findings. Rana (1983) and Dangi and Ameta (2005) tested acephate 0.05 per cent (750 $\mathrm{g} / \mathrm{ha}$ ) on okra was found most effective on the basis of per cent shoot and fruit infestation partially support the present findings.

The treatments of B. bassiana with 6.80 per cent infestation of shoot proved least effective followed by Btk (6.17\%). Lal (1997), Singh et al., (1998) and Awasthi et al., (2006) evaluated the bio-efficacy of $B$. thuringiensis and four insecticides against okra shoot and fruit borer and reported B. thuringiensis as less effective, conform the present findings.

The results were not corroborated with those of Sarode and Gahbane (1998), Patil et al., (2004) and Shinde et al., (2007) who reported neem seed kernel extract ineffective in controlling the shoot and fruit borer.

\section{Fruit damage by Earias spp.}

Based on the pooled date of Kharif 2013 and 2014, the treatment of indoxacarb (3.86 and $4.10 \%$ ), was found to be the most effective followed by spinosad (4.30 and 4.76\%), emamectin benzoate (4.64 and 5.08\%) and acetamiprid (5.16 and 5.30\%) damage both on number and weight basis, respectively.

Dhanalakshmi and Mallapur (2010) reported emamectin benzoate 5 SG @ 0.2 g/l (7.82\% damage) as most effective insecticide followed by spinosad $45 \mathrm{SC} @ 0.1 \mathrm{ml} / \mathrm{l}(9.9 \%$ damage) and indoxacarb 14.5 SC @ $0.3 \mathrm{ml} / \mathrm{l}$ (10.74\% damage) against fruit borer of okra, to some extent corroborate with the present findings.

Hirekurubar and Ambekar (2008) reported indoxacarb as the most effective treatment in reducing the okra shoot and fruit borer damage by registering 5.14 and $4.16 \%$ damage on number and weight basis, respectively support the present findings. 
Table.1 Bioefficacy of newer and biorational insecticides against shoot and fruit borer, Earias spp. on okra (kharif, 2013)

\begin{tabular}{|c|c|c|c|c|c|c|c|c|c|c|c|}
\hline \multirow{3}{*}{$\begin{array}{l}\text { S. } \\
\text { No. }\end{array}$} & \multirow[t]{3}{*}{ Treatments } & \multirow{3}{*}{$\begin{array}{l}\text { Conc. } \\
(\%) / \\
\text { Dosage }\end{array}$} & \multicolumn{9}{|c|}{ Mean per cent infestation of shoots after } \\
\hline & & & \multicolumn{4}{|c|}{ First spray } & \multicolumn{4}{|c|}{ Second spray } & \multirow[t]{2}{*}{ Mean } \\
\hline & & & 1 Day & 3 Day & 7 Day & 15 Day & 1 Day & 3 Day & 7 Day & 15 Day & \\
\hline \multirow[t]{2}{*}{1.} & \multirow[t]{2}{*}{ Acetamiprid $20 \mathrm{SP}$} & \multirow[t]{2}{*}{0.004} & 3.10 & 2.37 & 3.68 & 4.66 & 3.17 & 2.12 & 2.98 & 3.36 & 3.18 \\
\hline & & & $(10.14)$ & $(8.86)$ & $(11.06)$ & (12.47) & $(10.26)$ & $(8.37)$ & $(9.94)$ & $(10.56)$ & $(10.27)$ \\
\hline \multirow[t]{2}{*}{2.} & \multirow[t]{2}{*}{ Fipronil 5 SC } & \multirow[t]{2}{*}{0.01} & 1.86 & 0.95 & 2.00 & 3.10 & 2.02 & 1.52 & 1.77 & 2.00 & 3.78 \\
\hline & & & $(7.84)$ & $(5.59)$ & $(8.13)$ & (10.14) & $(8.17)$ & $(7.08)$ & $(7.65)$ & $(8.13)$ & $(11.20)$ \\
\hline \multirow[t]{2}{*}{3.} & \multirow[t]{2}{*}{ Acephate 75 SP } & \multirow[t]{2}{*}{0.037} & 4.00 & 3.23 & 4.13 & 5.73 & 4.64 & 3.54 & 3.81 & 4.73 & 4.23 \\
\hline & & & (11.54) & (10.35) & (11.73) & (13.85) & (12.44) & (10.84) & $(11.26)$ & (12.56) & (11.86) \\
\hline \multirow[t]{2}{*}{4.} & \multirow[t]{2}{*}{ NSKE } & \multirow[t]{2}{*}{5.0} & 5.11 & 4.36 & 4.84 & 6.79 & 5.77 & 4.38 & 4.49 & 5.91 & 5.21 \\
\hline & & & $(13.06)$ & (12.05) & $(12.71)$ & (15.10) & $(13.90)$ & $(12.08)$ & $(12.23)$ & $(14.07)$ & (13.19) \\
\hline \multirow[t]{2}{*}{5.} & \multirow[t]{2}{*}{$B t$ var. kurstaki } & \multirow[t]{2}{*}{$1 \mathrm{ml} \mathrm{l}^{-1}$} & 5.56 & 4.59 & 5.26 & 6.94 & 7.13 & 6.60 & 6.79 & 8.33 & 6.40 \\
\hline & & & (13.64) & (12.37) & (13.26) & (15.27) & (15.49) & (14.89) & (15.10) & (16.78) & (14.65) \\
\hline \multirow[t]{2}{*}{6.} & \multirow[t]{2}{*}{ Beauveria bassiana } & \multirow[t]{2}{*}{$1 \mathrm{~g} \mathrm{l}^{-1}$} & 6.00 & 5.13 & 5.73 & 7.18 & 7.31 & 6.69 & 7.04 & 8.69 & 6.72 \\
\hline & & & (14.18) & (13.09) & (13.85) & (15.54) & (15.69) & (14.99) & (15.39) & (17.14) & (15.03) \\
\hline \multirow[t]{2}{*}{7.} & \multirow[t]{2}{*}{ Indoxacarb $14.5 \mathrm{SC}$} & \multirow[t]{2}{*}{0.01} & 1.43 & 0.39 & 1.44 & 2.36 & 1.12 & 0.73 & 0.97 & 1.62 & 1.26 \\
\hline & & & $(6.87)$ & $(3.58)$ & $(6.89)$ & $(8.84)$ & $(6.07)$ & $(4.90)$ & $(5.65)$ & $(7.31)$ & $(6.44)$ \\
\hline \multirow[t]{2}{*}{8.} & \multirow{2}{*}{$\begin{array}{l}\text { Emamectin benzoate } 5 \\
\text { SG }\end{array}$} & $15 \mathrm{~g}$ a.i. & 2.72 & 1.57 & 2.67 & 4.22 & 2.12 & 1.66 & 2.12 & 2.47 & 2.44 \\
\hline & & $\mathrm{ha}^{-1}$ & $\begin{array}{l}(9.49) \\
\end{array}$ & $(7.20)$ & $(9.40)$ & (11.85) & $(8.37)$ & (7.40) & $(8.37)$ & (9.04) & (8.99) \\
\hline 9. & Spinosad 2.5 SC & 0.01 & 1.78 & 1.12 & 1.75 & 2.89 & 1.33 & 1.00 & 1.47 & 1.83 & 1.65 \\
\hline & & & $(7.67)$ & $(6.07)$ & $(7.60)$ & $(9.79)$ & $(6.62)$ & $(5.74)$ & $(6.96)$ & $(7.77)$ & $(7.37)$ \\
\hline 10. & Dimethoate $30 \mathrm{EC} /$ & $0.03 /$ & 4.81 & 3.70 & 4.87 & 6.00 & 4.87 & 3.75 & 4.97 & 5.14 & 4.76 \\
\hline & Malathion 50EC & 0.05 & (12.67) & (11.09) & (12.75) & (14.18) & (12.75) & (11.17) & $(12.88)$ & (13.10) & (12.61) \\
\hline 11. & Control (untreated) & & 7.11 & 7.50 & 8.16 & 8.72 & 8.14 & 8.26 & 8.54 & 9.41 & 8.23 \\
\hline & & & (15.46) & (15.89) & $(16.60)$ & $(17.18)$ & $(16.58)$ & $(16.70)$ & (16.99) & (17.86) & $(16.67)$ \\
\hline & S.Em. \pm & & 1.07 & 0.99 & 1.06 & 1.19 & 1.25 & 1.12 & 1.16 & 1.28 & 1.14 \\
\hline & $\mathrm{CD}(=0.05)$ & & 3.15 & 2.93 & 3.13 & 3.50 & 3.69 & 3.31 & 3.41 & 3.77 & 3.38 \\
\hline
\end{tabular}

* Mean of three replications; Figures in parentheses are angular transformed values

Table.2 Bioefficacy of newer and biorational insecticides against shoot and fruit borer, Earias spp. on okra (kharif, 2014)

\begin{tabular}{|c|c|c|c|c|c|c|c|c|c|c|c|}
\hline \multirow{3}{*}{$\begin{array}{l}\text { S. } \\
\text { No. }\end{array}$} & \multirow[t]{3}{*}{ Treatments } & \multirow{3}{*}{$\begin{array}{l}\text { Conc. } \\
(\%) / \\
\text { Dosage }\end{array}$} & \multicolumn{9}{|c|}{ Mean per cent infestation of shoots after } \\
\hline & & & \multicolumn{4}{|c|}{ First spray } & \multicolumn{4}{|c|}{$\begin{array}{l}\text { Second spray } \\
\end{array}$} & \multirow[t]{2}{*}{ Mean } \\
\hline & & & 1 Day & 3 Day & 7 Day & 15 Day & 1 Day & 3 Day & 7 Day & 15 Day & \\
\hline \multirow[t]{2}{*}{1.} & \multirow[t]{2}{*}{ Acetamiprid $20 \mathrm{SP}$} & \multirow[t]{2}{*}{0.004} & 3.87 & 2.80 & 3.58 & 4.00 & 3.00 & 2.80 & 2.98 & 3.54 & 3.32 \\
\hline & & & $(11.35)$ & $(9.63)$ & $(10.91)$ & (11.54) & (9.97) & $(9.63)$ & $(9.94)$ & $(10.84)$ & $(10.50)$ \\
\hline \multirow[t]{2}{*}{2.} & \multirow[t]{2}{*}{ Fipronil 5 SC } & \multirow[t]{2}{*}{0.01} & 2.18 & 1.46 & 2.38 & 3.11 & 2.11 & 1.43 & 1.96 & 2.87 & 2.19 \\
\hline & & & $(8.49)$ & (6.94) & $(8.87)$ & (10.16) & $(8.35)$ & $(6.87)$ & $(8.05)$ & $(9.75)$ & $(8.51)$ \\
\hline \multirow[t]{2}{*}{3.} & \multirow[t]{2}{*}{ Acephate $75 \mathrm{SP}$} & \multirow[t]{2}{*}{0.037} & 4.23 & 3.39 & 4.97 & 5.27 & 4.26 & 3.71 & 3.88 & 4.23 & 4.24 \\
\hline & & & $(11.87)$ & $(10.61)$ & $(12.88)$ & $(13.27)$ & $(11.91)$ & $(11.11)$ & $(11.36)$ & $(11.87)$ & (11.89) \\
\hline \multirow[t]{2}{*}{4.} & \multirow[t]{2}{*}{ NSKE } & \multirow[t]{2}{*}{5.0} & 5.43 & 4.32 & 4.53 & 6.12 & 5.25 & 4.96 & 5.66 & 6.54 & 5.35 \\
\hline & & & $(13.48)$ & $(12.00)$ & $(12.29)$ & $(14.32)$ & $(13.25)$ & $(12.87)$ & $(13.76)$ & $(14.82)$ & (13.38) \\
\hline \multirow[t]{2}{*}{5.} & \multirow[t]{2}{*}{ Bt var. kurstaki } & \multirow[t]{2}{*}{$1 \mathrm{ml} \mathrm{l}^{-1}$} & 5.75 & 4.65 & 4.94 & 7.25 & 6.00 & 5.22 & 6.44 & 7.20 & 5.93 \\
\hline & & & $(13.87)$ & $(12.45)$ & $(12.84)$ & $(15.62)$ & $(14.18)$ & $(13.21)$ & $(14.70)$ & $(15.56)$ & (14.10) \\
\hline \multirow[t]{2}{*}{6.} & \multirow[t]{2}{*}{ Beauveria bassiana } & \multirow[t]{2}{*}{$1 \mathrm{~g} \mathrm{l}^{-1}$} & 6.72 & 5.54 & 5.86 & 8.07 & 7.14 & 6.24 & 7.07 & 8.42 & 6.88 \\
\hline & & & $(15.02)$ & $(13.61)$ & $(14.01)$ & $(16.50)$ & $(15.50)$ & $(14.47)$ & $(15.42)$ & $(16.87)$ & $(15.21)$ \\
\hline \multirow[t]{2}{*}{7.} & \multirow[t]{2}{*}{ Indoxacarb 14.5 SC } & \multirow[t]{2}{*}{0.01} & 1.69 & 0.49 & 1.10 & 2.57 & 1.54 & 0.74 & 1.21 & 2.38 & 1.47 \\
\hline & & & $(7.47)$ & $(4.01)$ & $(6.02)$ & $(9.23)$ & $(7.13)$ & $(4.93)$ & $(6.32)$ & $(8.87)$ & $(6.95)$ \\
\hline \multirow[t]{2}{*}{8.} & \multirow{2}{*}{$\begin{array}{l}\text { Emamectin benzoate } 5 \\
\text { SG }\end{array}$} & 15 g a.i. & 3.07 & 2.44 & 3.19 & 3.67 & 2.66 & 1.69 & 1.78 & 3.00 & 2.69 \\
\hline & & $\mathrm{ha}^{-1}$ & $(10.09)$ & $(8.99)$ & $(10.29)$ & $(11.04)$ & $(9.39)$ & $(7.47)$ & (7.67) & $(9.97)$ & $(9.44)$ \\
\hline 9. & Spinosad 2.5 SC & 0.01 & 1.87 & 0.88 & 1.75 & 2.97 & 1.76 & 1.26 & 1.57 & 2.83 & 1.86 \\
\hline & & & $(7.86)$ & $(5.38)$ & $(7.60)$ & $(9.92)$ & $(7.62)$ & $(6.45)$ & $(7.20)$ & $(9.68)$ & $(7.84)$ \\
\hline 10. & Dimethoate $30 \mathrm{EC} /$ & $0.03 /$ & 5.00 & 4.13 & 4.99 & 5.82 & 4.95 & 3.87 & 4.82 & 5.30 & 4.86 \\
\hline & Malathion 50EC & 0.05 & $(12.92)$ & $(11.73)$ & $(12.91)$ & $(13.96)$ & $(12.86)$ & $(11.35)$ & $(12.68)$ & $(13.31)$ & (12.74) \\
\hline 11. & Control (untreated) & & 7.75 & 8.00 & 8.49 & 9.70 & 7.65 & 8.25 & 8.39 & 9.75 & 8.50 \\
\hline & & & $(16.16)$ & $(16.43)$ & $(16.94)$ & $(18.15)$ & $(16.06)$ & $(16.69)$ & $(16.84)$ & $(18.19)$ & $(16.95)$ \\
\hline & S.Em. \pm & & 1.13 & 1.03 & 1.07 & 1.25 & 1.16 & 1.09 & 1.16 & 1.27 & 1.15 \\
\hline & $\mathrm{CD}(=0.05)$ & & 3.34 & 3.05 & 3.17 & 3.68 & 3.41 & 3.22 & 3.41 & 3.74 & 3.38 \\
\hline
\end{tabular}

* Mean of three replications; Figures in parentheses are angular transformed values 
Table.3 Bioefficacy of newer and biorational insecticides against shoot and fruit borer, Earias spp. on okra (Pooled, kharif, 2013 and 2014)

\begin{tabular}{|c|c|c|c|c|c|c|c|c|c|c|c|}
\hline \multirow{3}{*}{$\begin{array}{l}\text { S. } \\
\text { No. }\end{array}$} & \multirow{3}{*}{ Treatments } & \multirow{3}{*}{$\begin{array}{c}\text { Conc. } \\
(\%) / \\
\text { Dosage }\end{array}$} & \multicolumn{9}{|c|}{ Mean per cent infestation of shoots after } \\
\hline & & & \multicolumn{4}{|c|}{ First spray } & \multicolumn{4}{|c|}{ Second spray } & \multirow[t]{2}{*}{ Mean } \\
\hline & & & 1 Day & 3 Day & 7 Day & 15 Day & 1 Day & 3 Day & 7 Day & 15 Day & \\
\hline \multirow[t]{2}{*}{1.} & \multirow{2}{*}{ Acetamiprid $20 \mathrm{SP}$} & \multirow{2}{*}{0.004} & 3.49 & 2.59 & 3.63 & 4.33 & 3.09 & 2.46 & 2.98 & 3.45 & 3.25 \\
\hline & & & $(10.76)$ & $(9.25)$ & $(10.98)$ & $(12.01)$ & $(10.12)$ & $(9.02)$ & $(9.94)$ & $(10.70)$ & $(10.39)$ \\
\hline \multirow[t]{2}{*}{2.} & \multirow{2}{*}{ Fipronil $5 \mathrm{SC}$} & \multirow{2}{*}{0.01} & 2.02 & 1.21 & 2.19 & 3.11 & 4.13 & 1.48 & 1.87 & 2.44 & 2.98 \\
\hline & & & $(8.17)$ & $(6.30)$ & $(8.51)$ & $(10.15)$ & $(11.73)$ & $(6.98)$ & $(7.85)$ & $(8.98)$ & $(9.94)$ \\
\hline \multirow[t]{2}{*}{3.} & \multirow[t]{2}{*}{ Acephate 75 SP } & \multirow[t]{2}{*}{0.037} & 4.12 & 3.31 & 4.55 & 5.50 & 4.45 & 3.63 & 3.85 & 4.48 & 4.23 \\
\hline & & & $(11.70)$ & $(10.48)$ & $(12.32)$ & $(13.56)$ & $(12.18)$ & $(10.98)$ & $(11.31)$ & $(12.22)$ & $(11.87)$ \\
\hline \multirow[t]{2}{*}{4.} & \multirow[t]{2}{*}{ NSKE } & \multirow[t]{2}{*}{5.0} & 5.27 & 4.34 & 4.69 & 6.46 & 5.51 & 4.67 & 5.08 & 6.23 & 5.28 \\
\hline & & & $(13.27)$ & $(12.02)$ & $(12.50)$ & $(14.72)$ & $(13.58)$ & $(12.48)$ & $(13.02)$ & $(14.45)$ & $(13.28)$ \\
\hline \multirow[t]{2}{*}{5.} & \multirow[t]{2}{*}{ Bt var. kurstaki } & \multirow[t]{2}{*}{$1 \mathrm{ml} \mathrm{l}^{-1}$} & 5.66 & 4.62 & 5.10 & 7.10 & 6.57 & 5.91 & 6.62 & 7.77 & 6.17 \\
\hline & & & $(13.76)$ & $(12.41)$ & $(13.05)$ & $(15.45)$ & $(14.85)$ & $(14.07)$ & $(14.90)$ & $(16.18)$ & $(14.38)$ \\
\hline \multirow[t]{2}{*}{6.} & \multirow[t]{2}{*}{ Beauveria bassiana } & \multirow[t]{2}{*}{$1 \mathrm{~g} \mathrm{l}^{-1}$} & 6.36 & 5.34 & 5.80 & 7.63 & 7.23 & 6.47 & 7.06 & 8.56 & 6.80 \\
\hline & & & $(14.61)$ & $(13.35)$ & $(13.93)$ & $(16.03)$ & $(15.59)$ & $(14.73)$ & $(15.40)$ & $(17.01)$ & $(15.12)$ \\
\hline \multirow[t]{2}{*}{7.} & \multirow[t]{2}{*}{ Indoxacarb 14.5 SC } & \multirow[t]{2}{*}{0.01} & 1.56 & 0.44 & 1.27 & 2.47 & 1.33 & 0.74 & 1.09 & 2.00 & 1.36 \\
\hline & & & $(7.17)$ & $(3.80)$ & $(6.47)$ & $(9.03)$ & $(6.62)$ & $(4.92)$ & $(5.99)$ & $(8.13)$ & $(6.70)$ \\
\hline \multirow[t]{2}{*}{8.} & \multirow{2}{*}{$\begin{array}{l}\text { Emamectin benzoate } 5 \\
\text { SG }\end{array}$} & $15 \mathrm{~g}$ a.i. & 2.90 & 2.01 & 2.93 & 3.95 & 2.39 & 1.68 & 1.95 & 2.74 & 2.57 \\
\hline & & $\mathrm{ha}^{-1}$ & $(9.80)$ & $(8.14)$ & $(9.86)$ & $(11.46)$ & $(8.89)$ & $(7.44)$ & $(8.03)$ & $(9.52)$ & $(9.22)$ \\
\hline 9. & Spinosad 2.5 SC & 0.01 & 1.83 & 1.00 & 1.75 & 2.93 & 1.55 & 1.13 & 1.52 & 2.33 & 1.75 \\
\hline & & & $(7.76)$ & $(5.74)$ & $(7.60)$ & $(9.86)$ & $(7.14)$ & $(6.10)$ & $(7.08)$ & $(8.78)$ & $(7.61)$ \\
\hline 10. & Dimethoate $\quad 30 \mathrm{EC} /$ & $0.03 /$ & 4.91 & 3.92 & 4.93 & 5.91 & 4.91 & 3.81 & 4.90 & 5.22 & 4.81 \\
\hline & Malathion 50EC & 0.05 & $(12.80)$ & $(11.41)$ & $(12.83)$ & $(14.07)$ & $(12.80)$ & $(11.26)$ & $(12.78)$ & $(13.21)$ & $(12.67)$ \\
\hline 11. & Control (untreated) & & 7.43 & 7.75 & 8.33 & 9.21 & 7.90 & 8.26 & 8.47 & 9.58 & 8.36 \\
\hline & & & $(15.82)$ & $(16.16)$ & $(16.77)$ & $(17.67)$ & $(16.32)$ & $(16.70)$ & $(16.91)$ & $(18.03)$ & $(16.81)$ \\
\hline & S.Em. \pm & & 1.10 & 1.01 & 1.07 & 1.22 & 1.21 & 1.11 & 1.16 & 1.27 & 1.15 \\
\hline & $\mathrm{CD}(=0.05)$ & & 3.25 & 2.99 & 3.15 & 3.59 & 3.56 & 3.26 & 3.41 & 3.75 & 3.38 \\
\hline
\end{tabular}

* Mean of three replications; Figures in parentheses are angular transformed values

Table.4 Bioefficacy of newer and biorational insecticides against shoot and fruit borer, Earias spp. on okra (Number basis) (kharif, 2013)

\begin{tabular}{|c|c|c|c|c|c|c|c|c|c|c|c|}
\hline \multirow{3}{*}{$\begin{array}{l}\text { S. } \\
\text { No. }\end{array}$} & \multirow[t]{3}{*}{ Treatments } & \multirow{3}{*}{$\begin{array}{l}\text { Conc. } \\
(\%) / \\
\text { Dosage }\end{array}$} & \multicolumn{9}{|c|}{ Mean per cent infestation of fruits after } \\
\hline & & & \multicolumn{4}{|c|}{ Second spray } & \multicolumn{4}{|c|}{ Third spray } & \multirow{2}{*}{ Mean } \\
\hline & & & 1 Day & 3 Day & 7 Day & 15 Day & 1 Day & 3 Day & 7 Day & 15 Day & \\
\hline \multirow[t]{2}{*}{1.} & \multirow[t]{2}{*}{ Acetamiprid $20 \mathrm{SP}$} & \multirow[t]{2}{*}{0.004} & 4.16 & 3.70 & 5.34 & 7.53 & 4.52 & 3.24 & 5.79 & 7.65 & 5.24 \\
\hline & & & $(11.77)$ & $(11.09)$ & (13.36) & $(15.93)$ & $(12.27)$ & $(10.37)$ & $(13.92)$ & $(16.06)$ & $(13.23)$ \\
\hline \multirow[t]{2}{*}{2.} & \multirow[t]{2}{*}{ Fipronil $5 \mathrm{SC}$} & \multirow[t]{2}{*}{0.01} & 5.72 & 4.26 & 6.64 & 7.90 & 5.29 & 4.11 & 6.80 & 8.00 & 6.09 \\
\hline & & & $(13.84)$ & $(11.91)$ & $(14.93)$ & $(16.32)$ & $(13.30)$ & $(11.70)$ & $(15.12)$ & $(16.43)$ & $(14.29)$ \\
\hline \multirow[t]{2}{*}{3.} & \multirow[t]{2}{*}{ Acephate 75 SP } & \multirow[t]{2}{*}{0.037} & 6.88 & 5.18 & 7.77 & 8.29 & 5.54 & 4.30 & 6.86 & 8.25 & 6.63 \\
\hline & & & $(15.21)$ & $(13.16)$ & (16.19) & $(16.73)$ & $(13.61)$ & $(11.97)$ & $(15.18)$ & $(16.69)$ & $(14.93)$ \\
\hline \multirow[t]{2}{*}{4.} & \multirow[t]{2}{*}{ NSKE } & \multirow[t]{2}{*}{5.0} & 7.49 & 6.33 & 8.92 & 9.24 & 6.35 & 5.20 & 6.97 & 8.43 & 7.37 \\
\hline & & & $(15.88)$ & $(14.57)$ & $(17.38)$ & $(17.70)$ & $(14.60)$ & (13.18) & $(15.31)$ & $(16.88)$ & $(15.75)$ \\
\hline \multirow[t]{2}{*}{5.} & \multirow[t]{2}{*}{$B t$ var. kurstaki } & \multirow[t]{2}{*}{$1 \mathrm{ml} \mathrm{l}^{-1}$} & 8.68 & 7.09 & 7.12 & 10.00 & 8.14 & 7.42 & 7.76 & 9.21 & 8.18 \\
\hline & & & (17.13) & $(15.44)$ & (15.48) & $(18.43)$ & $(16.58)$ & $(15.81)$ & $(16.17)$ & (17.67) & $(16.62)$ \\
\hline \multirow[t]{2}{*}{6.} & \multirow[t]{2}{*}{ Beauveria bassiana } & \multirow[t]{2}{*}{$1 \mathrm{~g} \mathrm{l}^{-1}$} & 9.17 & 8.00 & 8.30 & 10.41 & 9.00 & 8.15 & 8.43 & 9.76 & 8.90 \\
\hline & & & $(17.63)$ & $(16.43)$ & $(16.74)$ & $(18.82)$ & $(17.46)$ & $(16.59)$ & $(16.88)$ & $(18.20)$ & $(17.36)$ \\
\hline \multirow[t]{2}{*}{7.} & \multirow[t]{2}{*}{ Indoxacarb 14.5 SC } & \multirow[t]{2}{*}{0.01} & 3.15 & 2.08 & 4.78 & 5.04 & 3.43 & 2.66 & 5.40 & 6.23 & 4.10 \\
\hline & & & $(10.22)$ & $(8.29)$ & (12.63) & $(12.97)$ & $(10.67)$ & $(9.39)$ & $(13.44)$ & $(14.45)$ & $(11.68)$ \\
\hline \multirow[t]{2}{*}{8.} & \multirow{2}{*}{$\begin{array}{l}\text { Emamectin benzoate } \\
5 \mathrm{SG}\end{array}$} & $15 \mathrm{~g}$ a.i. & 3.98 & 3.00 & 5.10 & 6.74 & 4.19 & 3.76 & 5.63 & 7.02 & 4.93 \\
\hline & & $\mathrm{ha}^{-1}$ & $(11.51)$ & $(9.97)$ & $(13.05)$ & $(15.05)$ & $(11.81)$ & (11.18) & $(13.73)$ & $(15.36)$ & $(12.83)$ \\
\hline 9. & Spinosad 2.5 SC & 0.01 & 3.45 & 2.73 & 4.87 & 6.60 & 4.66 & 3.20 & 5.81 & 6.52 & 4.73 \\
\hline & & & $(10.70)$ & $(9.51)$ & $(12.75)$ & $(14.89)$ & $(12.47)$ & $(10.30)$ & $(13.95)$ & $(14.79)$ & $(12.56)$ \\
\hline 10. & Dimethoate $30 \mathrm{EC} /$ & $0.03 /$ & 7.17 & 6.20 & 8.88 & 9.00 & 6.20 & 5.00 & 6.66 & 8.23 & 7.17 \\
\hline & Malathion 50EC & 0.05 & $(15.53)$ & $(14.42)$ & (17.34) & $(17.46)$ & $(14.42)$ & $(12.92)$ & $(14.96)$ & $(16.67)$ & (15.53) \\
\hline 11. & Control (untreated) & & 10.96 & 12.34 & 13.65 & 15.27 & 11.23 & 12.14 & 14.60 & 16.55 & 13.34 \\
\hline & & & (19.33) & $(20.57)$ & $(21.68)$ & $(23.00)$ & $(19.58)$ & $(20.39)$ & $(22.46)$ & $(24.01)$ & $(21.42)$ \\
\hline & S.Em. \pm & & 0.84 & 0.82 & 0.86 & 0.96 & 0.86 & 0.82 & 0.87 & 0.94 & 0.87 \\
\hline & $\mathrm{CD}(=0.05)$ & & 2.50 & 2.45 & 2.56 & 2.84 & 2.58 & 2.44 & 2.61 & 2.78 & 2.59 \\
\hline
\end{tabular}

* Mean of three replications ; Figures in parentheses are angular transformed values 
Table.5 Bioefficacy of newer and biorational insecticides against shoot and fruit borer, Earias spp. on okra (Number basis) (kharif, 2014)

\begin{tabular}{|c|c|c|c|c|c|c|c|c|c|c|c|}
\hline \multirow{3}{*}{$\begin{array}{l}\text { S. } \\
\text { No. }\end{array}$} & \multirow[t]{3}{*}{ Treatments } & \multirow{3}{*}{$\begin{array}{c}\text { Conc. } \\
(\%) / \\
\text { Dosage }\end{array}$} & \multicolumn{9}{|c|}{ Mean per cent infestation of fruits after } \\
\hline & & & \multicolumn{4}{|c|}{ Second spray } & \multicolumn{4}{|c|}{ Third spray } & \multirow[t]{2}{*}{ Mean } \\
\hline & & & 1 Day & 3 Day & 7 Day & 15 Day & 1 Day & 3 Day & 7 Day & 15 Day & \\
\hline \multirow[t]{2}{*}{1.} & \multirow[t]{2}{*}{ Acetamiprid $20 \mathrm{SP}$} & \multirow[t]{2}{*}{0.004} & 4.45 & 3.90 & 5.25 & 5.60 & 4.46 & 3.40 & 6.42 & 7.12 & 5.08 \\
\hline & & & $(12.18)$ & $(11.39)$ & $(13.25)$ & (13.69) & $(12.19)$ & $(10.63)$ & $(14.68)$ & $(15.48)$ & $(13.02)$ \\
\hline \multirow[t]{2}{*}{2.} & \multirow[t]{2}{*}{ Fipronil $5 \mathrm{SC}$} & \multirow[t]{2}{*}{0.01} & 5.81 & 4.55 & 6.44 & 6.83 & 5.02 & 4.62 & 6.69 & 7.87 & 5.98 \\
\hline & & & (13.95) & $(12.32)$ & $(14.70)$ & $(15.15)$ & $(12.95)$ & $(12.41)$ & $(14.99)$ & $(16.29)$ & $(14.15)$ \\
\hline \multirow[t]{2}{*}{3.} & \multirow[t]{2}{*}{ Acephate 75 SP } & \multirow[t]{2}{*}{0.037} & 5.98 & 4.05 & 6.64 & 6.98 & 6.29 & 5.78 & 7.14 & 8.07 & 6.37 \\
\hline & & & $(14.15)$ & $(11.61)$ & $(14.93)$ & $(15.32)$ & $(14.52)$ & $(13.91)$ & $(15.50)$ & $(16.50)$ & $(14.61)$ \\
\hline \multirow[t]{2}{*}{4.} & \multirow[t]{2}{*}{ NSKE } & \multirow[t]{2}{*}{5.0} & 7.29 & 6.00 & 6.27 & 8.92 & 7.66 & 6.57 & 6.77 & 9.29 & 7.35 \\
\hline & & & $(15.66)$ & $(14.18)$ & $(14.50)$ & $(17.38)$ & $(16.07)$ & $(14.85)$ & $(15.08)$ & $(17.75)$ & $(15.73)$ \\
\hline \multirow[t]{2}{*}{5.} & \multirow[t]{2}{*}{ Bt var. kurstaki } & \multirow[t]{2}{*}{$1 \mathrm{ml} \mathrm{l}^{-1}$} & 8.26 & 7.12 & 7.90 & 9.56 & 8.12 & 7.45 & 7.84 & 10.36 & 8.33 \\
\hline & & & $(16.70)$ & $(15.48)$ & $(16.32)$ & $(18.01)$ & $(16.56)$ & $(15.84)$ & $(16.26)$ & $(18.78)$ & $(16.77)$ \\
\hline \multirow[t]{2}{*}{6.} & \multirow[t]{2}{*}{ Beauveria bassiana } & \multirow[t]{2}{*}{$1 \mathrm{~g} \mathrm{l}^{-1}$} & 9.11 & 8.46 & 8.98 & 10.22 & 9.37 & 8.40 & 8.97 & 11.42 & 9.37 \\
\hline & & & $(17.57)$ & $(16.91)$ & (17.44) & (18.64) & $(17.82)$ & $(16.85)$ & $(17.43)$ & (19.75) & $(17.82)$ \\
\hline \multirow[t]{2}{*}{7.} & \multirow[t]{2}{*}{ Indoxacarb 14.5 SC } & \multirow[t]{2}{*}{0.01} & 2.77 & 1.90 & 3.89 & 4.72 & 3.17 & 2.82 & 4.56 & 5.21 & 3.63 \\
\hline & & & $(9.58)$ & $(7.92)$ & $(11.38)$ & $(12.55)$ & $(10.26)$ & $(9.67)$ & $(12.33)$ & $(13.19)$ & $(10.98)$ \\
\hline \multirow[t]{2}{*}{8.} & \multirow{2}{*}{$\begin{array}{l}\text { Emamectin benzoate } \\
5 \mathrm{SG}\end{array}$} & $15 \mathrm{~g}$ a.i. & 3.59 & 2.96 & 4.59 & 5.02 & 4.00 & 3.19 & 5.08 & 6.37 & 4.35 \\
\hline & & $\mathrm{ha}^{-1}$ & $(10.92)$ & $(9.91)$ & $(12.37)$ & $(12.95)$ & (11.54) & $(10.29)$ & $(13.03)$ & $(14.62)$ & $(12.04)$ \\
\hline 9. & Spinosad 2.5 SC & 0.01 & 3.05 & 2.80 & 4.00 & 4.80 & 3.29 & 2.89 & 4.78 & 5.36 & 3.87 \\
\hline & & & $(10.06)$ & $(9.63)$ & $(11.54)$ & $(12.66)$ & $(10.45)$ & $(9.79)$ & $(12.63)$ & $(13.39)$ & $(11.35)$ \\
\hline 10. & Dimethoate $30 \mathrm{EC} /$ & $0.03 /$ & 6.25 & 5.09 & 7.72 & 8.00 & 6.56 & 5.85 & 7.74 & 8.59 & 6.98 \\
\hline & Malathion 50EC & 0.05 & $(14.48)$ & $(13.04)$ & $(16.13)$ & (16.43) & (14.84) & $(14.00)$ & (16.15) & $(17.04)$ & (15.31) \\
\hline 11. & Control (untreated) & & 12.16 & 13.67 & 15.25 & 17.45 & 12.41 & 14.79 & 16.62 & 18.28 & 15.08 \\
\hline & & & $(20.41)$ & $(21.70)$ & $(22.99)$ & $(24.69)$ & $(20.63)$ & $(22.62)$ & $(24.06)$ & $(25.31)$ & $(22.85)$ \\
\hline & S.Em. \pm & & 1.02 & 0.99 & 1.03 & 1.11 & 1.03 & 1.00 & 1.05 & 1.20 & 1.05 \\
\hline & $\mathrm{CD}(=0.05)$ & & 3.02 & 2.95 & 3.08 & 3.30 & 3.08 & 2.97 & 3.15 & 3.57 & 3.14 \\
\hline
\end{tabular}

* Mean of three replications; Figures in parentheses are angular transformed values

Table.6 Bioefficacy of newer and biorational insecticides against shoot and fruit borer, Earias spp. infesting okra (Number basis) (Pooled, kharif, 2013 and 2014)

\begin{tabular}{|c|c|c|c|c|c|c|c|c|c|c|c|}
\hline \multirow{3}{*}{$\begin{array}{l}\text { S. } \\
\text { No. }\end{array}$} & \multirow[t]{3}{*}{ Treatments } & \multirow{3}{*}{$\begin{array}{c}\text { Conc. } \\
(\%) / \\
\text { Dosage }\end{array}$} & \multicolumn{9}{|c|}{ Mean per cent infestation of fruits after } \\
\hline & & & \multicolumn{4}{|c|}{ Second spray } & \multicolumn{4}{|c|}{ Third spray } & \multirow[t]{2}{*}{ Mean } \\
\hline & & & 1 Day & 3 Day & 7 Day & 15 Day & 1 Day & 3 Day & 7 Day & 15 Day & \\
\hline \multirow[t]{2}{*}{1.} & \multirow[t]{2}{*}{ Acetamiprid $20 \mathrm{SP}$} & \multirow[t]{2}{*}{0.004} & 4.31 & 3.80 & 5.30 & 6.57 & 4.49 & 3.32 & 6.11 & 7.39 & 5.16 \\
\hline & & & $(11.98)$ & $(11.24)$ & $(13.30)$ & $(14.85)$ & $(12.23)$ & $(10.50)$ & $(14.30)$ & $(15.77)$ & $(13.13)$ \\
\hline \multirow[t]{2}{*}{2.} & \multirow[t]{2}{*}{ Fipronil $5 \mathrm{SC}$} & \multirow[t]{2}{*}{0.01} & 5.77 & 4.41 & 6.54 & 7.37 & 5.16 & 4.37 & 6.75 & 7.94 & 6.03 \\
\hline & & & $(13.89)$ & $(12.12)$ & $(14.82)$ & $(15.75)$ & $(13.12)$ & $(12.06)$ & $(15.05)$ & $(16.36)$ & $(14.22)$ \\
\hline \multirow[t]{2}{*}{3.} & \multirow[t]{2}{*}{ Acephate 75 SP } & \multirow[t]{2}{*}{0.037} & 6.43 & 4.62 & 7.21 & 7.64 & 5.92 & 5.04 & 7.00 & 8.16 & 6.50 \\
\hline & & & $(14.69)$ & $(12.41)$ & $(15.57)$ & $(16.04)$ & $(14.08)$ & $(12.97)$ & $(15.34)$ & $(16.60)$ & $(14.77)$ \\
\hline \multirow[t]{2}{*}{4.} & \multirow[t]{2}{*}{ NSKE } & \multirow[t]{2}{*}{5.0} & 7.39 & 6.17 & 7.60 & 9.08 & 7.01 & 5.89 & 6.87 & 8.86 & 7.36 \\
\hline & & & $(15.77)$ & $(14.38)$ & $(16.00)$ & $(17.54)$ & $(15.35)$ & $(14.04)$ & $(15.20)$ & $(17.32)$ & (15.74) \\
\hline \multirow[t]{2}{*}{5.} & \multirow[t]{2}{*}{ Bt var. kurstaki } & \multirow[t]{2}{*}{$1 \mathrm{ml} \mathrm{l}^{-1}$} & 8.47 & 7.11 & 7.51 & 9.78 & 8.13 & 7.44 & 7.80 & 9.79 & 8.25 \\
\hline & & & $(16.92)$ & $(15.46)$ & $(15.91)$ & $(18.22)$ & $(16.57)$ & $(15.82)$ & $(16.22)$ & $(18.23)$ & $(16.69)$ \\
\hline \multirow[t]{2}{*}{6.} & \multirow[t]{2}{*}{ Beauveria bassiana } & \multirow[t]{2}{*}{$1 \mathrm{~g} \mathrm{l}^{-1}$} & 9.14 & 8.23 & 8.64 & 10.32 & 9.19 & 8.28 & 8.70 & 10.59 & 9.13 \\
\hline & & & $(17.60)$ & $(16.67)$ & $(17.09)$ & $(18.73)$ & $(17.64)$ & $(16.72)$ & $(17.15)$ & $(18.99)$ & $(17.59)$ \\
\hline \multirow[t]{2}{*}{7.} & \multirow[t]{2}{*}{ Indoxacarb 14.5 SC } & \multirow[t]{2}{*}{0.01} & 2.96 & 1.99 & 4.34 & 4.88 & 3.30 & 2.74 & 4.98 & 5.72 & 3.86 \\
\hline & & & $(9.91)$ & $(8.11)$ & $(12.02)$ & (12.76) & $(10.47)$ & $(9.53)$ & (12.89) & $(13.84)$ & (11.34) \\
\hline \multirow[t]{2}{*}{8.} & \multirow{2}{*}{$\begin{array}{l}\text { Emamectin benzoate } \\
5 \mathrm{SG}\end{array}$} & $15 \mathrm{~g}$ a.i. & 3.79 & 2.98 & 4.85 & 5.88 & 4.10 & 3.48 & 5.36 & 6.70 & 4.64 \\
\hline & & $\mathrm{ha}^{-1}$ & $(11.22)$ & $(9.94)$ & $(12.72)$ & $(14.03)$ & $(11.68)$ & $(10.74)$ & $(13.38)$ & $(15.00)$ & $(12.44)$ \\
\hline 9. & Spinosad 2.5 SC & 0.01 & 3.25 & 2.77 & 4.44 & 5.70 & 3.98 & 3.05 & 5.30 & 5.94 & 4.30 \\
\hline & & & (10.39) & $(9.57)$ & (12.16) & $(13.81)$ & $(11.50)$ & $(10.05)$ & $(13.30)$ & $(14.11)$ & (11.97) \\
\hline 10. & Dimethoate $30 \mathrm{EC} /$ & $0.03 /$ & 6.71 & 5.65 & 8.30 & 8.50 & 6.38 & 5.43 & 7.20 & 8.41 & 7.07 \\
\hline & Malathion 50EC & 0.05 & $(15.01)$ & $(13.74)$ & $(16.74)$ & $(16.95)$ & $(14.63)$ & $(13.47)$ & $(15.56)$ & $(16.86)$ & $(15.42)$ \\
\hline 11. & Control (untreated) & & 11.56 & 13.01 & 14.45 & 16.36 & 11.82 & 13.47 & 15.61 & 17.42 & 14.21 \\
\hline & & & (19.88) & (21.14) & (22.34) & (23.86) & (20.11) & (21.53) & (23.27) & (24.67) & $(22.15)$ \\
\hline & S.Em. \pm & & 0.93 & 0.90 & 0.95 & 1.03 & 0.95 & 0.91 & 0.96 & 1.07 & 0.96 \\
\hline & $\mathrm{CD}(=0.05)$ & & 2.76 & 2.70 & 2.82 & 3.07 & 2.83 & 2.70 & 2.88 & 3.17 & 2.87 \\
\hline
\end{tabular}

* Mean of three replications; Figures in parentheses are angular transformed values 
Table.7 Bioefficacy of newer and biorational insecticides against shoot and fruit borer, Earias spp. on okra (Weight basis) kharif, 2013

\begin{tabular}{|c|c|c|c|c|c|c|c|c|c|c|c|}
\hline \multirow{3}{*}{$\begin{array}{l}\text { S. } \\
\text { No. }\end{array}$} & \multirow[t]{3}{*}{ Treatments } & \multirow{3}{*}{$\begin{array}{c}\text { Conc. } \\
(\%) / \\
\text { Dosage }\end{array}$} & \multicolumn{9}{|c|}{ Mean per cent infestation of fruits after } \\
\hline & & & \multicolumn{4}{|c|}{ Second spray } & \multicolumn{4}{|c|}{ Third spray } & \multirow[t]{2}{*}{ Mean } \\
\hline & & & 1 Day & 3 Day & 7 Day & 15 Day & 1 Day & 3 Day & 7 Day & 15 Day & \\
\hline \multirow[t]{2}{*}{1.} & \multirow{2}{*}{ Acetamiprid $20 \mathrm{SP}$} & \multirow{2}{*}{0.004} & 4.00 & 3.52 & 5.14 & 7.10 & 4.33 & 3.11 & 5.42 & 7.12 & 4.97 \\
\hline & & & $(11.54)$ & $(10.81)$ & $(13.10)$ & $(15.45)$ & (12.01) & $(10.16)$ & $(13.46)$ & $(15.48)$ & (12.88) \\
\hline \multirow[t]{2}{*}{2.} & \multirow{2}{*}{ Fipronil $5 \mathrm{SC}$} & \multirow{2}{*}{0.01} & 5.61 & $\begin{array}{l}4.12 \\
\end{array}$ & 6.52 & 7.76 & 5.17 & 3.82 & 6.44 & 7.70 & 5.89 \\
\hline & & & $(13.70)$ & (11.71) & (14.79) & (16.17) & (13.14) & $(11.27)$ & $(14.70)$ & $(16.11)$ & $(14.05)$ \\
\hline \multirow[t]{2}{*}{3.} & \multirow[t]{2}{*}{ Acephate 75 SP } & \multirow[t]{2}{*}{0.037} & 6.72 & 5.00 & 7.61 & 8.02 & 5.22 & 4.15 & 6.39 & 8.00 & 6.39 \\
\hline & & & $(15.02)$ & $(12.92)$ & $(16.01)$ & $(16.45)$ & (13.21) & (11.75) & (14.64) & (16.43) & (14.64) \\
\hline \multirow[t]{2}{*}{4.} & \multirow[t]{2}{*}{ NSKE } & \multirow[t]{2}{*}{5.0} & 7.36 & 6.82 & 7.00 & 9.32 & 6.14 & 5.00 & 6.72 & 8.04 & 7.05 \\
\hline & & & $(15.74)$ & $(15.14)$ & (15.34) & (17.78) & (14.35) & $(12.92)$ & $(15.02)$ & $(16.47)$ & $(15.40)$ \\
\hline \multirow[t]{2}{*}{5.} & \multirow[t]{2}{*}{ Bt var. kurstaki } & \multirow[t]{2}{*}{$1 \mathrm{ml} \mathrm{l}^{-1}$} & 8.22 & 6.90 & 6.94 & 9.62 & 7.95 & 7.06 & 7.20 & 8.34 & 7.78 \\
\hline & & & $(16.66)$ & $(15.23)$ & $(15.27)$ & (18.07) & (16.38) & $(15.41)$ & $(15.56)$ & (16.79) & $(16.19)$ \\
\hline \multirow[t]{2}{*}{6.} & \multirow[t]{2}{*}{ Beauveria bassiana } & \multirow[t]{2}{*}{$1 \mathrm{~g} \mathrm{l}^{-1}$} & 9.00 & 7.82 & 8.03 & 10.05 & 8.72 & 8.00 & 8.11 & 9.03 & 8.60 \\
\hline & & & $(17.46)$ & $(16.24)$ & $(16.46)$ & (18.48) & (17.18) & $(16.43)$ & $(16.55)$ & $(17.49)$ & $(17.05)$ \\
\hline \multirow[t]{2}{*}{7.} & \multirow[t]{2}{*}{ Indoxacarb 14.5 SC } & \multirow[t]{2}{*}{0.01} & 3.00 & 1.95 & 4.66 & 5.00 & 3.31 & 2.52 & 5.21 & 6.00 & 3.96 \\
\hline & & & $(9.97)$ & $(8.03)$ & $(12.47)$ & $(12.92)$ & $(10.48)$ & $(9.13)$ & $(13.19)$ & (14.18) & (11.47) \\
\hline \multirow[t]{2}{*}{8.} & \multirow{2}{*}{$\begin{array}{l}\text { Emamectin benzoate } 5 \\
\text { SG }\end{array}$} & $15 \mathrm{~g}$ a.i. & 3.84 & 2.90 & 5.00 & 6.65 & 4.00 & 3.59 & 5.22 & 6.62 & 4.73 \\
\hline & & ha $^{-1}$ & $(11.30)$ & $(9.80)$ & $(12.92)$ & $(14.94)$ & (11.54) & $(10.92)$ & $(13.21)$ & $(14.91)$ & (12.56) \\
\hline 9. & Spinosad 2.5 SC & 0.01 & 3.34 & 2.68 & 4.63 & 6.45 & 4.52 & 3.07 & 5.70 & 6.34 & 4.59 \\
\hline & & & $(10.53)$ & $(9.42)$ & $(12.43)$ & $(14.71)$ & $(12.27)$ & $(10.09)$ & $(13.81)$ & $(14.58)$ & $(12.37)$ \\
\hline 10. & Dimethoate $\quad 30 \mathrm{EC} /$ & $0.03 /$ & 7.04 & 6.06 & 8.64 & 8.80 & 6.00 & 4.81 & 6.49 & 8.00 & 6.98 \\
\hline & Malathion 50EC & & $(15.39)$ & $(14.25)$ & $(17.09)$ & $(17.26)$ & $(14.18)$ & $(12.67)$ & $(14.76)$ & $(16.43)$ & $(15.32)$ \\
\hline 11. & Control (untreated) & & 10.10 & 12.00 & 13.16 & 14.29 & 10.25 & 11.35 & 13.00 & 14.50 & 12.33 \\
\hline & & & $(18.53)$ & $(20.27)$ & $(21.27)$ & $(22.21)$ & $(18.67)$ & $(19.69)$ & $(21.13)$ & $(22.38)$ & $(20.56)$ \\
\hline & S.Em. \pm & & 0.85 & 0.81 & 0.83 & 0.93 & 0.84 & 0.79 & 0.84 & 0.89 & 0.85 \\
\hline & $\mathrm{CD}(=0.05)$ & & 2.52 & 2.41 & 2.48 & 2.75 & 2.51 & 2.37 & 2.51 & 2.64 & 2.52 \\
\hline
\end{tabular}

* Mean of three replications; Figures in parentheses are angular transformed values

Table.8 Bioefficacy of newer and biorational insecticides against shoot and fruit borer, Earias spp. on okra (Weight basis) kharif, 2014

\begin{tabular}{|c|c|c|c|c|c|c|c|c|c|c|c|}
\hline \multirow{3}{*}{$\begin{array}{l}\text { S. } \\
\text { No. }\end{array}$} & \multirow[t]{3}{*}{ Treatments } & \multirow{3}{*}{$\begin{array}{l}\text { Conc. } \\
(\%) / \\
\text { Dosage }\end{array}$} & \multicolumn{9}{|c|}{ Mean per cent infestation of fruits after } \\
\hline & & & \multicolumn{4}{|c|}{ Second spray } & \multicolumn{4}{|c|}{$\begin{array}{r}\text { Third spray } \\
\end{array}$} & \multirow[t]{2}{*}{ Mean } \\
\hline & & & 1 Day & 3 Day & 7 Day & 15 Day & 1 Day & 3 Day & 7 Day & 15 Day & \\
\hline \multirow[t]{2}{*}{1.} & \multirow[t]{2}{*}{ Acetamiprid 20 SP } & \multirow[t]{2}{*}{0.004} & 5.09 & 4.26 & 6.29 & 7.44 & 4.70 & 3.95 & 6.07 & 7.20 & 5.63 \\
\hline & & & $(13.04)$ & $(11.91)$ & $(14.52)$ & $(15.83)$ & $(12.52)$ & $(11.46)$ & $(14.26)$ & $(15.56)$ & $(13.72)$ \\
\hline \multirow[t]{2}{*}{2.} & \multirow[t]{2}{*}{ Fipronil $5 \mathrm{SC}$} & \multirow[t]{2}{*}{0.01} & 6.35 & 5.27 & 7.45 & 8.82 & 6.11 & 5.05 & 7.31 & 8.55 & 6.86 \\
\hline & & & $(14.60)$ & $(13.27)$ & $(15.84)$ & $(17.28)$ & $(14.31)$ & $(12.99)$ & $(15.69)$ & $(17.00)$ & $(15.19)$ \\
\hline \multirow[t]{2}{*}{3.} & \multirow[t]{2}{*}{ Acephate $75 \mathrm{SP}$} & \multirow[t]{2}{*}{0.037} & 7.00 & 6.73 & 7.89 & 8.75 & 6.90 & 6.62 & 7.73 & 8.65 & 7.53 \\
\hline & & & $(15.34)$ & $(15.04)$ & $(16.31)$ & $(17.21)$ & $(15.23)$ & $(14.91)$ & $(16.14)$ & $(17.10)$ & $(15.93)$ \\
\hline \multirow[t]{2}{*}{4.} & \multirow[t]{2}{*}{ NSKE } & \multirow[t]{2}{*}{5.0} & 8.94 & 7.20 & 7.64 & 10.27 & 8.74 & 7.00 & 7.06 & 9.91 & 8.35 \\
\hline & & & $(17.40)$ & $(15.56)$ & $(16.05)$ & $(18.69)$ & $(17.20)$ & $(15.34)$ & $(15.41)$ & $(18.35)$ & $(16.79)$ \\
\hline \multirow[t]{2}{*}{5.} & \multirow[t]{2}{*}{$B t$ var. kurstaki } & \multirow[t]{2}{*}{$1 \mathrm{ml} \mathrm{l}^{-1}$} & 9.00 & 8.75 & 8.83 & 10.76 & 8.94 & 8.65 & 8.69 & 10.57 & 9.27 \\
\hline & & & $(17.46)$ & $(17.21)$ & $(17.29)$ & $(19.15)$ & $(17.40)$ & $(17.10)$ & $(17.14)$ & $(18.97)$ & $(17.73)$ \\
\hline \multirow[t]{2}{*}{6.} & \multirow[t]{2}{*}{ Beauveria bassiana } & \multirow[t]{2}{*}{$1 \mathrm{~g} \mathrm{l}^{-1}$} & 10.00 & 9.75 & 9.90 & 11.25 & 9.47 & 8.80 & 9.81 & 10.89 & 9.98 \\
\hline & & & $(18.43)$ & $(18.19)$ & $(18.34)$ & $(19.60)$ & $(17.92)$ & $(17.26)$ & $(18.25)$ & $(19.27)$ & $(18.42)$ \\
\hline \multirow[t]{2}{*}{7.} & \multirow[t]{2}{*}{ Indoxacarb 14.5 SC } & \multirow[t]{2}{*}{0.01} & 3.59 & 2.86 & 4.79 & 6.16 & 3.46 & 2.69 & 4.56 & 5.80 & 4.24 \\
\hline & & & $(10.92)$ & $(9.74)$ & $(12.64)$ & $(14.37)$ & $(10.72)$ & $(9.44)$ & $(12.33)$ & (13.94) & $(11.88)$ \\
\hline \multirow[t]{2}{*}{8.} & \multirow[t]{2}{*}{ Emamectin benzoate $5 \mathrm{SG}$} & $15 \mathrm{~g}$ a.i. & 4.70 & 4.05 & 6.17 & 7.23 & 4.58 & 3.86 & 5.94 & 7.00 & 5.44 \\
\hline & & $\mathrm{ha}^{-1}$ & $(12.52)$ & $(11.61)$ & $(14.38)$ & $(15.60)$ & $(12.36)$ & $(11.33)$ & $(14.11)$ & $(15.34)$ & $(13.49)$ \\
\hline 9. & Spinosad 2.5 SC & 0.01 & 4.19 & 3.90 & 5.15 & 6.78 & 4.02 & 3.74 & 5.00 & 6.67 & 4.93 \\
\hline & & & (11.81) & (11.39) & (13.12) & (15.09) & (11.57) & (11.15) & (12.92) & (14.97) & (12.83) \\
\hline 10. & Dimethoate & $0.03 /$ & 8.21 & 7.64 & 8.22 & 9.67 & 8.00 & 7.56 & 8.10 & 9.34 & 8.34 \\
\hline & Malathion 50EC & 0.05 & (16.65) & $(16.05)$ & (16.66) & (18.12) & $(16.43)$ & (15.96) & $(16.54)$ & $(17.80)$ & $(16.79)$ \\
\hline 11. & Control (untreated) & & 11.23 & 12.80 & 14.21 & 16.62 & 11.25 & 12.10 & 13.00 & 15.87 & 13.39 \\
\hline & & & (19.58) & (20.96) & $(22.15)$ & (24.06) & (19.60) & (20.36) & (21.13) & (23.48) & (21.46) \\
\hline & S.Em. \pm & & 1.04 & 1.03 & 1.06 & 1.13 & 1.01 & 0.98 & 1.04 & 1.09 & 1.05 \\
\hline & $\mathrm{CD}(=0.05)$ & & 3.08 & 3.08 & 3.15 & 3.36 & 3.02 & 2.91 & 3.13 & 3.23 & 3.12 \\
\hline
\end{tabular}

* Mean of three replications; Figures in parentheses are angular transformed values 
Table.9 Bioefficacy of newer and biorational insecticides against shoot and fruit borer, Earias spp. on okra (Weight basis) (Pooled, kharif, 2013 and 2014)

\begin{tabular}{|c|c|c|c|c|c|c|c|c|c|c|c|}
\hline \multirow{3}{*}{$\begin{array}{l}\text { S. } \\
\text { No. }\end{array}$} & \multirow[t]{3}{*}{ Treatments } & \multirow{3}{*}{$\begin{array}{l}\text { Conc. } \\
(\%) / \\
\text { Dosage }\end{array}$} & \multicolumn{9}{|c|}{ Mean per cent infestation of fruits after } \\
\hline & & & \multicolumn{4}{|c|}{ Second spray } & \multicolumn{4}{|c|}{ Third spray } & \multirow[t]{2}{*}{ Mean } \\
\hline & & & 1 Day & 3 Day & 7 Day & 15 Day & 1 Day & 3 Day & 7 Day & 15 Day & \\
\hline \multirow[t]{2}{*}{1.} & \multirow[t]{2}{*}{ Acetamiprid $20 \mathrm{SP}$} & \multirow[t]{2}{*}{0.004} & 4.55 & 3.89 & 5.72 & 7.27 & 4.52 & 3.53 & 5.75 & 7.16 & 5.30 \\
\hline & & & $(12.31)$ & $(11.38)$ & (13.83) & (15.64) & $(12.27)$ & $(10.83)$ & $(13.87)$ & $(15.52)$ & $(13.31)$ \\
\hline \multirow[t]{2}{*}{2.} & \multirow[t]{2}{*}{ Fipronil $5 \mathrm{SC}$} & \multirow[t]{2}{*}{0.01} & 5.98 & 4.70 & 6.99 & 8.29 & 5.64 & 4.44 & 6.88 & 8.13 & 6.38 \\
\hline & & & $(14.15)$ & $(12.51)$ & $(15.32)$ & $(16.73)$ & $(13.74)$ & $(12.16)$ & $(15.20)$ & $(16.56)$ & $(14.63)$ \\
\hline \multirow[t]{2}{*}{3 . } & \multirow[t]{2}{*}{ Acephate $75 \mathrm{SP}$} & \multirow[t]{2}{*}{0.037} & 6.86 & 5.87 & 7.75 & 8.39 & 6.06 & 5.39 & 7.06 & 8.33 & 6.96 \\
\hline & & & $(15.18)$ & $(14.02)$ & $(16.16)$ & $(16.83)$ & $(14.25)$ & $(13.42)$ & $(15.41)$ & $(16.77)$ & $(15.30)$ \\
\hline \multirow[t]{2}{*}{4.} & \multirow[t]{2}{*}{ NSKE } & \multirow[t]{2}{*}{5.0} & 8.15 & 7.01 & 7.32 & 9.80 & 7.44 & 6.00 & 6.89 & 8.98 & 7.70 \\
\hline & & & $(16.59)$ & $(15.35)$ & (15.70) & $(18.24)$ & $(15.83)$ & (14.18) & $(15.22)$ & $(17.43)$ & $(16.11)$ \\
\hline \multirow[t]{2}{*}{5.} & \multirow[t]{2}{*}{ Bt var. kurstaki } & \multirow[t]{2}{*}{$1 \mathrm{ml} \mathrm{l}^{-1}$} & 8.61 & 7.83 & 7.89 & 10.19 & 8.45 & 7.86 & 7.95 & 9.46 & 8.53 \\
\hline & & & (17.06) & $(16.24)$ & $(16.31)$ & $(18.62)$ & (16.89) & $(16.28)$ & $(16.37)$ & $(17.91)$ & $(16.98)$ \\
\hline \multirow[t]{2}{*}{6.} & \multirow[t]{2}{*}{ Beauveria bassiana } & \multirow[t]{2}{*}{$1 \mathrm{~g} \mathrm{l}^{-1}$} & 9.50 & 8.79 & 8.97 & 10.65 & 9.10 & 8.40 & 8.96 & 9.96 & 9.29 \\
\hline & & & $(17.95)$ & $(17.24)$ & $(17.42)$ & (19.05) & $(17.55)$ & $(16.85)$ & $(17.42)$ & $(18.40)$ & $(17.75)$ \\
\hline \multirow[t]{2}{*}{7.} & \multirow[t]{2}{*}{ Indoxacarb 14.5 SC } & \multirow[t]{2}{*}{0.01} & 3.30 & 2.41 & 4.73 & 5.58 & 3.39 & 2.61 & 4.89 & 5.90 & 4.10 \\
\hline & & & $(10.46)$ & $(8.92)$ & $(12.55)$ & $(13.66)$ & $(10.60)$ & $(9.29)$ & $(12.77)$ & $(14.06)$ & $(11.68)$ \\
\hline \multirow[t]{2}{*}{8.} & \multirow{2}{*}{$\begin{array}{l}\text { Emamectin benzoate } 5 \\
\text { SG }\end{array}$} & $15 \mathrm{~g}$ a.i. & 4.27 & 3.48 & 5.59 & 6.94 & 4.29 & 3.73 & 5.58 & 6.81 & 5.08 \\
\hline & & $\mathrm{ha}^{-1}$ & $(11.93)$ & $(10.74)$ & $(13.67)$ & $(15.27)$ & $(11.95)$ & $(11.13)$ & (13.66) & $(15.13)$ & $(13.03)$ \\
\hline 9. & Spinosad 2.5 SC & 0.01 & 3.77 & 3.29 & 4.89 & 6.62 & 4.27 & 3.41 & 5.35 & 6.51 & 4.76 \\
\hline & & & $(11.19)$ & $(10.45)$ & $(12.78)$ & $(14.90)$ & $(11.93)$ & $(10.63)$ & $(13.37)$ & $(14.78)$ & $(12.60)$ \\
\hline 10. & Dimethoate $\quad 30 \mathrm{EC} /$ & $0.03 /$ & 7.63 & 6.85 & 8.43 & 9.24 & 7.00 & 6.19 & 7.30 & 8.67 & 7.66 \\
\hline & Malathion 50EC & 0.05 & $(16.03)$ & (15.17) & $(16.88)$ & $(17.69)$ & (15.34) & (14.40) & (15.67) & (17.12) & $(16.07)$ \\
\hline 11. & Control (untreated) & & 10.67 & 12.40 & 13.69 & 15.46 & 10.75 & 11.73 & 13.00 & 15.19 & 12.86 \\
\hline & & & (19.06) & (20.62) & (21.71) & $(23.15)$ & (19.14) & (20.02) & $(21.13)$ & (22.93) & $(21.01)$ \\
\hline & S.Em. \pm & & 0.94 & 0.92 & 0.94 & 1.03 & 0.93 & 0.89 & 0.94 & 0.99 & 0.95 \\
\hline & $\mathrm{CD}(=0.05)$ & & 2.80 & 2.74 & 2.81 & 3.06 & 2.77 & 2.64 & 2.82 & 2.93 & 2.82 \\
\hline
\end{tabular}

* Mean of three replications; Figures in parentheses are angular transformed values

The treatments of fipronil (6.03 and $6.38 \%)$, acephate $(6.50$ and $6.96 \%)$, dimethoate $(7.07$ and $7.66 \%)$ and NSKE (7.36 and 7.77\%) damage both on number and weight basis, respectively existed in moderate group of efficacy (Tables 3-9). Singh et al., (2009) applied three spray of acephate 75 SP @ 1500 $\mathrm{g}$ a.i./ha against $E$. vittella and recorded minimum fruit damage 13.96 per cent partially conform the present findings. Rana (1983) and Dangi and Ameta (2005) tested acephate 0.05 per cent $(750 \mathrm{~g} / \mathrm{ha})$ on okra was found most effective on the basis of per cent shoot and fruit infestation, partially support the present findings. Shinde et al., (2007) recorded minimum fruit infestation by $E$. vittella with the application of neem seed powder@ $@ 50 \mathrm{~kg}$ a.i./ha, partially support the present findings.

The treatments of $B$. bassiana (9.13 and 9.29\%) damage proved least effective followed by Btk (8.25 and $8.53 \%$ ) on number and weight basis, respectively. Kumawat (1997) found Btk less effective against shoot and fruit borer corroborate with the present findings.

\section{References}

Anonymous, 2015. Horticultural Statistics at a Glance 2015. National Horticulture Board, pp. 152 and 204.

Awasthi, A. K., Bisen, R. K., Choure, M.K. and Pandya, K. S. 2006. Bio-efficacy of Bacillus thuringiensis formulation and some insecticides against shoot and fruit borer, Earias vitella (Fab.) on okra. Journal of Insect Science, 13(1-2): 5556.

Dabhi, M. V., Acharya, M. F., Koshiya, D. J. and Khanpara, A. V. 2012. Bio-efficacy of various insecticides against okra shoot and fruit borer, Earias vittella (Fab.). AGRES- An International $e$ Journal, 1(1): 65-70. 
Dangi, P. R. and Ameta, O. P. 2005. Incidence of okra fruit borer, E. vittella. National Conference on Applied Entomology, Sept. 26-28, 2005 held at Rajasthan College of Agriculture, Udaipur, and pp. 84-85.

Das, S. N., Subhrajyoti, R., Chatterjee, M. L. and Ray, S. 2001. Bio-efficacy, yield, benefit and cost effectivity of some new molecules against okra fruit borer, Earias vittella (F.); (Noctuidae: Lepidoptera). Journal of Interacademicia, 5(3): 346-351.

Dhanalakshmi, D. N. and Mallapur, C. P. 2010. Evaluation of new promising molecules against fruit borers in okra. International Journal of Plant Protection, 3(2): 268-270.

Dhawan, A. K. and Sidhu, A. S. 1984. Incidence and relative abundance of different species of spotted bollworm on okra at Ludhiana. Punjab Journal of Research, PAU, 21(4): 533-542.

Gadekar, S. D. 2013. Seasonal incidence and management of major sucking pests of okra, Abelmoschus esculentus (L.) Moench. Thesis, submitted to Swami Keshwanand Rajasthan Agricultural University, Bikaner (Raj.).

Hirekurubar, R. B. and Ambekar, J. S. 2008. Bio-efficacy of newer insecticides against shoot and fruit borer of okra and their impact on natural enemies. Crop Research, 36(3): 302-307.

Kumawat, R. L. 1997. Seasonal incidence and insecticidal control of shoot and fruit borer on okra (Abelmoschus esculentus). M.Sc. Thesis submitted to Rajasthan Agricultural University, Bikaner.

Lal, R. L. 1997. Seasonal incidence and insecticidal control of major insect pests of okra (Abelmoschus esculentus (L.) Moench). M.Sc. (Ag.). Thesis, submitted to Rajasthan Agricultural University, campus- Jobner.
Manjanaik, C., Herle, P. S. and Singh, G. K. 2002. Chemical control of fruit borer, Earias spp. in coastal zone of Karnataka, Environmental Science of Ecology, 20 (3): 692-695.

Naik, N. R. and Kumar, A. 2014. Efficacy of certain insecticides and seasonal incidence of shoot and fruit borer, Earias vittella Fab. On okra. Annals of Plant Protection Sciences, 22(1): 95-97.

Pareek, B. L., Sharma, G. R. and Bhatanagar, K. N. 1986. Seasonal incidence of major insect pests of okra in semi-arid region of Rajasthan. Annals of Arid Zone, 25 (3): 222-224.

Pareek, B. L. and Bhargava, M. C. 2003. Estimation of avoidable losses in vegetable crops caused by borers under semi-arid conditions of Rajasthan. Insect Environment, 9: 59-60.

Parmar, K. D., Korat, D. M., Joshi, M. N., Patel, A. R. and Shah 2013. Relative bio-efficacy of insecticides/mitiecides against pest complex of okra. Karnataka Journal of Agricultural Science, 26(3): 375-378.

Parthiban, P., Baskaran, R.K and Thangavel, K. 2014. Field efficacy of Emamectin benzoate 5 WC against lepidopteran pests of okra. Annals of Plant Protection Sciences, 22(1): 98-102.

Patra, S., Chatterjee, M. L. and Arunava, S. 2009. Field efficacy of insecticides for management of Earias vittella in okra. Annals of Plant Protection Sciences, 17(2): 471-472.

Patil, S. B., Udikeri, S. S. and Khadi, B. M. 2004. Thiamethoxam 35 FS- a new seed dresser formulation for sucking pest control in cotton crop. Pestology, 28(3): 34-36.

Raghuraman, M., Ajanta Birah and Gupta, G.P. 2008. Bio efficacy of acetamiprid on sucking pests in cotton. Indian Journal of Entomology, 70(4):319-325.

Rana, B. S. 1983. Studies on the effectiveness 
of some insecticides against insect pests of Bhindi, Abelmoschus esculentus (L.) Moench, M.Sc. Thesis, submitted to Mohan Lal Sukhadia University, Udaipur.

Rao, S. and Rajendran, R. 2003, Joint action potential of neem with other plant extracts against the leafhopper Amrasca devastans (Distant) on okra. Pest Management and Economic Zoology, 10: 131-136.

Samuthiravelu, P. and David, B. V. 1991. Bioefficacy of neem oil and endosulfan against fruit borer, E. vittella (Fab.) (Noctuidae: Lepidoptera) on okra. Madras Agriculture Journal, 78 (1-4): 77-78.

Sarode, S. V. and Gabhane, A. T. 1998. Effect of neem and insecticide combinations for okra fruit borer management. Pest Management in Horticultural Ecosystem, 4(1): 54-56.

Shah, B. R.; Vyas, H. N. and Jhala, R. C. 2001. Population dynamics of major insect pests of okra, Abelmoschus esculentus (L.) Moench in relation to weather parameters. National Conference on Plant Protection-New Horizons in the Millennium held at Rajasthan College of Agriculture Udaipur from Feb., 23-25, 2001: pp.4.
Shinde, B. D., Sarkate, M. B., Memade, P. W. and Sable, Y. R. 2007. Bioefficacy of botanical microbial and synthetic insecticides against okra fruit borer. Pestology, 31(3): 19.22.

Shinde, S. T., Shetgar, S. S. and Badgujar, A. G. 2011. Bio-efficacy of different insecticides against major pests of okra. Journal of Entomological Research, 35 (2): 133-137.

Singh, G., Bhardwaj, S. C. and Dhaliwal, G. S. 1998. Evaluation of some biopesticides for the management of fruit borer, Earias spp. On okra crop. Indian Journal of Ecology, 25(2): 187-189.

Singh, S., Choudhary, D. P., Sharma, H. C., Mahala, R. S. and Mathur, Y. S. 2009b. Management of shoot and fruit borer Earias spp. In okra. Indian Journal of Applied Entomology, 23(2): 130-131.

Shinde, B. D., Sarkate, M. B., Memade, P. W. and Sable, Y. R. 2007. Bioefficacy of botanical microbial and synthetic insecticides against okra fruit borer. Pestology, 31(3): 19.22.

Zala, S. P., Patel, J. R. and Patel, N. C. 1999. Impact of weather on magnitude of Earias vittella (Fab.) infesting okra. Indian Journal of Entomology, 61 (4): 351-355

\section{How to cite this article:}

Surendra Kumar Yadav, K.C. Kumawat, H.L. Deshwal, Susheel Kumar and Manohar S.V.S. 2017. Bioefficacy of Newer and Biorational Insecticides against Shoot and Fruit Borer, Earias spp. on Okra. Int.J.Curr.Microbiol.App.Sci. 6(7): 1035-1044.

doi: https://doi.org/10.20546/ijcmas.2017.607.124 